\title{
Neural Network Backstepping Controller Design for Fractional-Order Nonlinear Systems
}

\author{
Youjun Chen $\mathbb{D}^{1}$ and Songyu Wang $\mathbb{D}^{2}$ \\ ${ }^{1}$ School of Mathematics and Statistics, North China University of Water Resources and Electric Power, Zhengzhou 450046, China \\ ${ }^{2}$ School of Information and Management Sciences, Henan Agricultural University, Zhengzhou 450046, China
}

Correspondence should be addressed to Youjun Chen; chenyoujun@ncwu.edu.cn

Received 18 April 2021; Accepted 8 June 2021; Published 21 June 2021

Academic Editor: Heng Liu

Copyright (C) 2021 Youjun Chen and Songyu Wang. This is an open access article distributed under the Creative Commons Attribution License, which permits unrestricted use, distribution, and reproduction in any medium, provided the original work is properly cited.

\begin{abstract}
In this work, a backstepping controller design for fractional-order strict feedback systems is investigated and the neural network control method is used. It is noted that in the standard backstepping design, the fractional derivative of the virtual quantity needs to be calculated repeatedly, which will lead to a sharp increase in the number of controller terms with the increase of the system dimension and finally make the control system difficult to bear. To handle the estimation error, certain robust terms in the controller at the last step are designed. The stability of the controlled system is proven strictly. In addition, the proposed controller has a simple form which can be easily implemented. Finally, in order to verify our theoretical method, the control simulation based on a fractional-order chaotic system is implemented.
\end{abstract}

\section{Introduction}

Backstepping design is a recursive design method. It is a systematic design method for systems with uncertain parameters first proposed by Kanellakopoulos et al. in 1991 [1]. Its main ideas of this control method are to obtain a feedback controller by recursively constructing Lyapunov function of closed-loop system, select proper control law by deriving the Lyapunov function along the trajectory of closed-loop system, ensure the boundedness of closedloop system trajectory, and guarantee the convergence of the tracking error. The backstepping design method is suitable for both linear and nonlinear systems, so it has been widely used as soon as proposed. In addition, the backstepping method can be used together with many other control methods, such as sliding mode control [2, 3], adaptive fuzzy control (AFC) $[4,5]$, and intermittent control [6], and it is very effective in controlling the strict feedback systems. Backstepping control has some advantages, such as global stability, easy design, and implementation of the controller. However, it also has a big weakness named "explosion of terms," which is caused by repeatedly deriving the virtual controller. In fact, it is difficult to solve this problem completely. Meanwhile, it is well known that system uncertainties exist in most realworld systems. Hence, it is meaningful but difficult to investigate the backstepping control of uncertain nonlinear systems avoiding explosion of terms.

On the contrary, fractional calculus has almost the same history as the integer one. However, due to the complexity of the theory and the lack of corresponding physical background, it has not been developed as it should be. In 1983, Mandelbrot pointed out that there are a large number of fractional dimension phenomena in nature, and thus, fractional calculus has rapidly become a research hotspot. Studies show that many physical systems exhibit fractional-order dynamic behavior due to their special material and chemical properties, which are called fractional-order systems. Using fractional-order models to describe objects with fractional-order characteristics can better reveal the essential characteristics and behavior of objects [7]. Fractional calculus is the extension of integer calculus, and integral calculus is a special case of fractional calculus, so it is more universal to study 
fractional-order systems [8-10]. Compared with the integer-order model, the fractional-order model has clearer physical meaning and more concise expression in describing complex physical and mechanical problems; fractional-order control expands the degree of freedom of control and can obtain better control performance; fractional-order calculus has memory performance which ensures the influence of historical information on the present and future, and is conducive to improving the quality of control [11-14]. In the field of integer-order systems, based on Lyapunov stability theory, the control of nonlinear systems has been widely studied and a series of results have been obtained. However, due to the late start of fractional-order control and the complexity of the theory, the fractional-order stability theory and controller design method are far less developed than the control of integer-order systems.

In the last several decades, the nonlinear system control based on universal function approximators (neural networks and fuzzy system) has received much attention $[4,14-18]$. It is shown that universal function approximators show an excellent performance in approximating system uncertainties. Thus, backstepping control based on universal function approximators is an interesting research idea. In this aspect, many prior works have been given. In [4], fuzzy backstepping controller was designed for fractional-order systems, where the fractional virtual inputs are approximated by fuzzy systems. In [19], optimal backstepping control implemented for fractional-order systems was investigated. In [20], fuzzy backstepping control of strict-feedback fractional-order uncertain nonlinear systems was studied, and some interesting results were given. In [5], by using optimal control, type-2 fuzzy control, and sliding mode control, backstepping control of fractional systems is addressed, and it is shown that the backstepping control can be well mixed with many other control strategies. In [21], fuzzy backstepping control of fractional-order systems is addressed, and the saturation phenomenon in fractional systems was also studied. In [22], to avoid the chattering phenomenon in the traditional backstepping control, a novel control method was developed. Some recent works can be referred to [23-28] and the references therein.

The key idea of this paper is to try to solve the problem of "term explosion" in the backstepping control of fractional-order systems. Backstepping control technique is employed in the controller design. It should be emphasized that no prior knowledge about the system uncertainties and the unknown part of the fractional-order system is needed, and the term explosion problem is also solved by using neural networks to approximate the system uncertainties.

\section{Preliminaries}

2.1. Problem Description. In this paper, we will consider the fractional-order nonlinear systems listed as

$$
\left\{\begin{array}{l}
D_{t}^{\alpha} \zeta_{1}(t)=f_{1}\left(\bar{\zeta}_{1}(t)\right)+\zeta_{2}(t), \\
D_{t}^{\alpha} \zeta_{2}(t)=f_{2}\left(\bar{\zeta}_{2}(t)\right)+\zeta_{3}(t), \\
\vdots \\
D_{t}^{\alpha} \zeta_{n}(t)=f_{n}\left(\bar{\zeta}_{n}(t)\right)+u(t), \\
y(t)=\zeta_{1}(t),
\end{array}\right.
$$

with $\bar{\zeta}_{i}=\left[\zeta_{1}, \ldots, \zeta_{i}\right]^{T} \in R^{i}, i=1, \ldots, n$ being the state, $y(t)=\zeta_{1}(t)$ being the output, $f_{i}\left(\bar{\zeta}_{i}\right), i=1, \ldots, n$ being unknown functions, and $u(t)$ representing the input. Apparently, system (1) represents a large class of strict feedback fractional-order systems.

Our aim is to design a proper controller such that the system output $y(t)$ tracks the desired trajectory $\zeta_{d}(t)$ with certain accuracy. Since $f_{i}\left(\bar{\zeta}_{i}\right)$ is assumed to be unknown, it can be approximated by neural networks, which will be introduced later in this section. In addition, to meet our objective, we need the following assumptions.

Assumption 1. The referenced function $\zeta_{d}$ is smooth and is known in advance.

Assumption 2. The signal $\bar{\zeta}$ is always known.

2.2. Neural Network Description. In this part, we will give a brief description of a multilayer network that will be used later. The structure of the used neural networks is depicted in Figure 1.

In real-world applications, a neural network is represented in the following form:

$$
y_{k}\left(s, w_{k}\right)=\sum_{j=1}^{h} \omega_{k j} \varphi_{k j}\left(\sum_{i=1}^{n} v_{j i} s_{i}+\theta_{j}\right)=w_{k}^{T} \psi_{k}(\cdot),
$$

where $w_{k}=\left[\begin{array}{c}\omega_{k 1} \\ \vdots \\ \omega_{k h}\end{array}\right], \psi_{k}=\left[\begin{array}{c}\varphi_{k 1}\left(\sum_{i=1}^{n} v_{1 i} s_{i}+\theta_{1}\right) \\ \vdots \\ \varphi_{k h}\left(\sum_{i=1}^{n} v_{h i} s_{i}+\theta_{h}\right)\end{array}\right], n, h$, and $m$ are the number of neurons in input layer, hidden layer, and output layer, respectively, $y_{k}$ denotes the output, and $v_{j i}$ is selected randomly on the interval $[-1,1]$. According to [29-31], $\varphi(\cdot)$ is chosen as

$$
\varphi(x)=\frac{e^{x}-e^{-x}}{e^{x}+e^{-x}}
$$

Thus, we have

$$
y=W^{T} \psi(\cdot),
$$

with $W=\left[\begin{array}{c}w_{1}^{T} \\ w_{2}^{T} \\ \vdots \\ w_{m}^{T}\end{array}\right]$ and $\psi(\cdot)=\left[\begin{array}{cccc}\psi_{1}(\cdot) & 0 & \ldots & 0 \\ 0 & \psi_{2}(\cdot) & \ldots & 0 \\ \vdots & \vdots & \ddots & \vdots \\ 0 & 0 & \ldots & \psi_{m}(\cdot)\end{array}\right]$. 


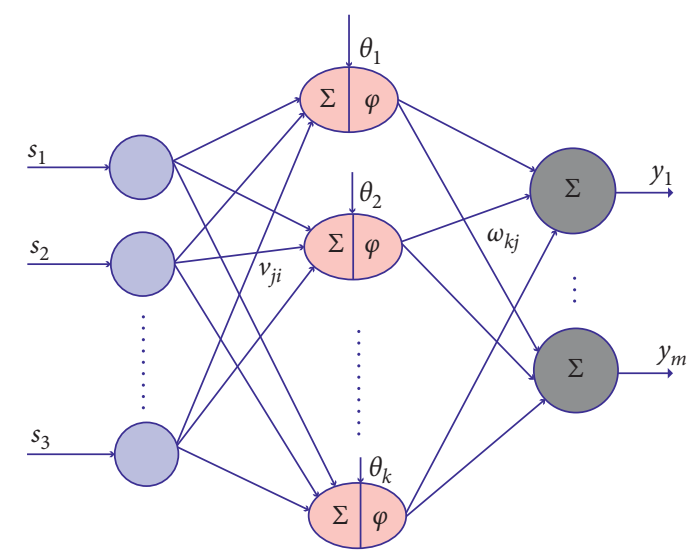

Figure 1: Structure of a neural network.

Generally, we can used the above neural network to estimate a nonlinear function as

$$
f(\zeta(t))=W^{* T}(t) \psi(\zeta(t))+\varepsilon(\zeta(t)),
$$

with $\varepsilon(\zeta(t))$ being the optimal approximation error vector. $W^{*}$ is defined by

$$
W^{*}=\arg \min _{W}[\sup |\widehat{f}(\zeta(t))-f(\zeta(t))|] .
$$

To facilitate the controller design, we need the following assumption.

Assumption 3. We can find some $\bar{\varepsilon}_{i}$ so that $\varepsilon_{i} \leq \bar{\varepsilon}_{i}$, with $i=1,2, \ldots, m$.

2.3. Fractional Calculus. In this paper, the Caputo fractional-order derivative will be used, which is expressed by

$$
{ }_{0}^{C} D_{t}^{\alpha} f(t)=\frac{1}{\Gamma(1-\alpha)} \int_{0}^{t}(t-\tau)^{-\alpha} f^{\prime}(\tau) \mathrm{d} \tau,
$$

with $0<\alpha \leq 1$ being the order and $\Gamma(\cdot)$ representing Euler's function. In the following, we will use $D_{t}^{\alpha}$ to replace ${ }_{0}^{C} D_{t}^{\alpha}$ for convenience. used.

The following results about the fractional calculus will be

Lemma 1 (see [8]). Suppose that $z(t)=0$ is an equilibrium of

$$
D_{t}^{\alpha} z(t)=f(z(t))
$$

One can find a Lyapunov function $V(t, z(t))$ and three class- $k$ functions $\varrho_{i}(t), i=1,2,3$, such that

$$
\begin{aligned}
& \varrho_{1}(\|z(t)\|) \leq V(t, z(t)) \leq \varrho_{2}(\|z(t)\|), \\
& D_{t}^{\beta} V(t, z(t)) \leq-\varrho_{3}(\|z(t)\|) .
\end{aligned}
$$

Then, $z(t)=0$ is asymptotical stability.

Lemma 2 (see [21]). Suppose that $z(t) \in \mathscr{R}^{n}$ is a smooth function. The following inequality holds:

$$
\frac{1}{2} D_{t}^{\alpha} z^{T}(t) z(t) \leq x^{T}(t) D_{t}^{\alpha} z(t)
$$

\section{Main Results}

In this part, the controller design together with the stability analysis will be given based the backstepping design procedure. To meet the control aim, let

$$
\left\{\begin{array}{l}
z_{1}(t)=\zeta_{1}(t)-\zeta_{d}(t), \\
z_{i}(t)=\zeta_{i}(t)-\alpha_{i-1}(t), \quad i=2, \ldots, n,
\end{array}\right.
$$

with $\alpha_{i}$ being a virtual input to be designed. The controller can be designed recursively because in the traditional backstepping design, virtual input $\alpha_{i+1}(t)$ depends on $\alpha_{i}(t)$ and its derivative. Thus, the whole design process can be divided into $n$ steps.

Step 1. According to the first equation in (11), we can obtain $D_{t}^{\alpha} z_{1}(t)=f\left(\bar{\zeta}_{1}(t)\right)-D_{t}^{\alpha} \zeta_{d}(t)+\zeta_{2}(t)=\gamma_{1}\left(\bar{\zeta}_{1}(t)\right)+\zeta_{2}(t)$,

with $\gamma_{1}\left(\bar{\zeta}_{1}(t)\right)=f\left(\bar{\zeta}_{1}(t)\right)-D_{t}^{\alpha} \zeta_{d}(t)$ representing an unknown function to be approximated. Then, $\gamma_{1}\left(\bar{\zeta}_{1}(t)\right)$ can be approximated using neural network (5) as

$$
\widehat{\gamma}_{1}\left(\bar{\zeta}_{1}(t), W_{1}(t)\right)=W_{1}^{T}(t) \psi\left(\bar{\zeta}_{1}(t)\right)
$$

Denote

$$
W^{*}=\arg \min _{W_{1}(t)}\left[\sup \left|\gamma\left(\bar{\zeta}_{1}(t)\right)-\widehat{\gamma}\left(\bar{\zeta}_{1}(t), W_{1}(t)\right)\right|\right] \text {. }
$$

It should be emphasized that $W_{1}^{*}$ is a constant vector whose exact value is not needed in the controller design. Let

$$
\begin{gathered}
\widetilde{W_{1}}(t)=W_{1}(t)-W_{1}^{*}, \\
\varepsilon_{1}\left(\bar{\zeta}_{1}(t)\right)=\gamma\left(\bar{\zeta}_{1}(t)\right)-\widehat{\gamma}\left(\bar{\zeta}_{1}(t), W_{1}^{*}\right),
\end{gathered}
$$

where $\widehat{\gamma}\left(\bar{\zeta}_{1}(t), W_{1}^{*}\right)=W_{1}^{*} \psi\left(\bar{\zeta}_{1}(t)\right)$. Like that, in the literature [32-34], it is reasonable to assume that $\bar{\zeta}_{1}(t)$ is bounded, i.e., $\left|\varepsilon_{1}\left(\bar{\zeta}_{1}(t)\right)\right|<\bar{\varepsilon}_{1}$ with $\bar{\varepsilon}_{1}$ being an unknown constant. Thus, it is easy to obtain that

$$
\begin{aligned}
& \hat{\gamma}\left(\bar{\zeta}_{1}(t), W_{1}(t)\right)-\gamma\left(\bar{\zeta}_{1}(t)\right) \\
& \quad=\widehat{\gamma}\left(\bar{\zeta}_{1}(t), W_{1}(t)\right)-\widehat{\gamma}\left(\bar{\zeta}_{1}(t), W_{1}^{*}\right)+\widehat{\gamma}\left(\bar{\zeta}_{1}(t), W_{1}^{*}\right)-\gamma\left(\bar{\zeta}_{1}(t)\right) .
\end{aligned}
$$

Using (16), (17) can be written as

$$
\begin{aligned}
& \widehat{\gamma}\left(\bar{\zeta}_{1}(t), W_{1}(t)\right)-\widehat{\gamma}\left(\bar{\zeta}_{1}(t), W_{1}^{*}\right)-\varepsilon_{1}\left(\bar{\zeta}_{1}(t)\right) \\
& =\widetilde{W}_{1}(t)^{T} \psi\left(\bar{\zeta}_{1}(t)\right)-\varepsilon_{1}\left(\bar{\zeta}_{1}(t)\right) .
\end{aligned}
$$

Substituting (15) and (18) into (17), we have

$$
\widehat{\gamma}\left(\bar{\zeta}_{1}(t), W_{1}(t)\right)-\gamma\left(\bar{\zeta}_{1}(t)\right)=\widetilde{W}_{1}(t)^{T} \psi\left(\bar{\zeta}_{1}(t)\right)-\varepsilon_{1}\left(\bar{\zeta}_{1}(t)\right) \text {. }
$$

Thus, the virtual control $\alpha_{1}$ is given by 


$$
\alpha_{1}(t)=-W_{1}^{T}(t) \psi\left(\bar{\zeta}_{1}(t)\right)-k_{1} z_{1}(t)
$$

Step 2. $i, 2 \leq i \leq n-1$. We can design

with $k_{1}>0$ being a constant.

$$
\alpha_{i}(t)=-\widehat{\gamma}_{i}(t)-z_{i-1}(t)-k_{i} z_{i}(t)=-W_{i}^{T}(t) \psi\left(\bar{\zeta}_{i}(t)\right)-z_{i-1}(t)-k_{i} z_{i}(t)
$$

with $\widehat{\gamma}_{i}(t)$ being the estimation of

$$
\gamma_{i}\left(\bar{\zeta}_{i}(t)\right)=f_{i}\left(\bar{\zeta}_{i}(t)\right)-\sum_{j=1}^{n-1}\left(\frac{\partial \alpha_{i-1}(t)}{\partial \zeta_{j}(t)} D_{t}^{\alpha} \zeta_{j}(t)+\frac{\partial \alpha_{i-1}(t)}{\partial \zeta_{d}^{(j-1)}(t)} \zeta_{d}^{(j)}(t)\right) .
$$

Step $n$. At this last step, we can construct the following controller:

$$
u(t)=-W_{n}^{T}(t) \psi\left(\bar{\zeta}_{n}(t)\right)-z_{n-1}(t)-k_{n} z_{n}(t)+u_{c}(t),
$$

with $u_{c}$ being a supervisory input which is used to handle the approximation errors of neural networks. To meet the control aim, the robust control term is given by

$$
u_{c}(t)=-\sum_{j=1}^{n} k_{1 j}\left|z_{j}(t)\right|
$$

with $k_{1 j}>0, j=1,2, \ldots, n$ being proper design parameters satisfying certain conditions (see, the conditions in Theorem 1).
In the controller design, neural networks' parameters are adjusted as

$$
D_{t}^{\alpha} \widehat{W}_{i}(t)=\lambda_{i} z_{i}(t) \psi\left(\bar{\zeta}_{i}(t)\right)
$$

with $\lambda_{i}>0$ being a design parameter.

The following theorem is presented to show the controlled system's stability.

Theorem 1. Consider system (1) satisfying Assumptions 1-3. If the virtual inputs are given by (20) and (21) and the final controller is given by (23) with a robust term (24), then the tracking error $z_{1}$ converges to origin asymptotically, and all signals remain bounded in the control process.

Consider a Lyapunov function as

$$
V_{1}=\frac{1}{2} z_{1}^{2}(t)+\frac{1}{2 \lambda_{1}} \tilde{W}_{1}^{T}(t) \tilde{W}_{1}(t)
$$

Noting that $\zeta_{2}(t)=z_{2}(t)+\alpha_{1}(t)$, by using Lemma 2 and using (1), (11), and (20), we have

$$
\begin{aligned}
D_{t}^{\alpha} V_{1}(t) & \leq z_{1}(t) D_{t}^{\alpha} z_{1}(t)+\frac{1}{\lambda_{1}} \widetilde{W}_{1}^{T}(t) D_{t}^{\alpha} W_{1}(t) \\
& =z_{1}(t)\left[f_{1}\left(\bar{\zeta}_{1}(t)\right)+\zeta_{2}(t)-D_{t}^{\alpha} \zeta_{d}(t)\right]+\frac{1}{\lambda_{1}} \widetilde{W}_{1}^{T}(t) D_{t}^{\alpha} W_{1}(t) \\
& =z_{1}(t)\left[f_{1}\left(\bar{\zeta}_{1}(t)\right)+z_{2}(t)+\alpha_{1}(t)-D_{t}^{\alpha} \zeta_{d}(t)\right]+\frac{1}{\lambda_{1}} \widetilde{W}_{1}^{T}(t) D_{t}^{\alpha} W_{1}(t) \\
& =z_{1}(t)\left[-W_{1}^{T}(t) \psi\left(\bar{\zeta}_{1}(t)\right)-k_{1} z_{1}(t)+f_{1}\left(\bar{\zeta}_{1}(t)\right)+z_{2}(t)-D_{t}^{\alpha} \zeta_{d}(t)\right]+\frac{1}{\lambda_{1}} \widetilde{W}_{1}^{T}(t) D_{t}^{\alpha} W_{1}(t) \\
& =z_{1}(t)\left[\gamma_{1}\left(\bar{\zeta}_{1}(t)\right)-W_{1}^{T}(t) \psi\left(\bar{\zeta}_{1}(t)\right)-k_{1} z_{1}(t)+z_{2}(t)\right]+\frac{1}{\lambda_{1}} \tilde{W}_{1}^{T}(t) D_{t}^{\alpha} W_{1}(t) \\
& =z_{1}(t)\left[\widetilde{W}_{1}(t)^{T} \psi\left(\bar{\zeta}_{1}(t)\right)-\varepsilon_{1}\left(\bar{\zeta}_{1}(t)\right)-k_{1} z_{1}(t)+z_{2}(t)\right]+\frac{1}{\lambda_{1}} \widetilde{W}_{1}^{T}(t) D_{t}^{\alpha} W_{1}(t) .
\end{aligned}
$$

Substituting (25) into (27), we have

$D_{t}^{\alpha} V_{1}(t) \leq-z_{1}(t) \varepsilon_{1}\left(\bar{\zeta}_{1}(t)\right)-k_{1} z_{1}^{2}(t)+z_{1}(t) z_{2}(t)$.

Next, let the Lyapunov function be

$$
V_{i}(t)=V_{1}(t)+\frac{1}{2} \sum_{j=2}^{i} z_{j}^{2}(t)+\frac{1}{2 \lambda_{j}} \sum_{j=2}^{i} \tilde{W}_{j}^{T}(t) \tilde{W}_{j}(t)
$$

According to (1), (11), (20), (28), and Lemma 2, we have 


$$
\begin{aligned}
D_{t}^{\alpha} V_{n-1} \leq & -z_{1}(t) \varepsilon_{1}\left(\bar{\zeta}_{1}(t)\right)-k_{1} z_{1}^{2}(t)+z_{1}(t) z_{2}(t)+\sum_{j=2}^{n-1} z_{j}(t) D_{t}^{\alpha} z_{j}(t)+\frac{1}{\lambda_{j}} \sum_{j=2}^{n-1} \widetilde{W}_{j}^{T}(t) D_{t}^{\alpha} W_{j}(t) \\
= & \sum_{j=2}^{n-1} z_{j}(t)\left[f_{j}\left(\bar{\zeta}_{j}(t)\right)+z_{j+1}(t)+\alpha_{j}(t)-\sum_{l=2}^{j}\left(\frac{\partial \alpha_{j-1}(t)}{\partial \zeta_{l}(t)} D_{t}^{\alpha} \zeta_{l}(t)+\frac{\partial \alpha_{j-1}(t)}{\partial \zeta_{d}^{(l-1)}(t)} \zeta_{d}^{(l)}(t)\right)\right] \\
& -z_{1}(t) \varepsilon_{1}\left(\bar{\zeta}_{1}(t)\right)-k_{1} z_{1}^{2}(t)+z_{1}(t) z_{2}(t)+\frac{1}{\lambda_{j}} \sum_{j=2}^{n-1} \widetilde{W}_{j}^{T}(t) D_{t}^{\alpha} W_{j}(t) \\
= & \sum_{j=2}^{n-1} z_{j}(t)\left[z_{j+1}(t)+\alpha_{j}(t)+\gamma_{j}\left(\bar{\zeta}_{j}(t)\right)\right]-z_{1}(t) \varepsilon_{1}\left(\bar{\zeta}_{1}(t)\right)-k_{1} z_{1}^{2}(t)+z_{1}(t) z_{2}(t)+\frac{1}{\lambda_{j}} \sum_{j=2}^{n-1} \widetilde{W}_{j}^{T}(t) D_{t}^{\alpha} W_{j}(t) .
\end{aligned}
$$

Substituting (21) into (30) and using (25), we have

$$
\begin{aligned}
D_{t}^{\alpha} V_{n-1} \leq & \sum_{j=2}^{n-1} z_{j}(t)\left[z_{j+1}(t)+\alpha_{j}(t)+\gamma_{j}\left(\bar{\zeta}_{j}(t)\right)\right]-z_{1}(t) \varepsilon_{1}\left(\bar{\zeta}_{1}(t)\right)-k_{1} z_{1}^{2}(t)+z_{1}(t) z_{2}(t)+\frac{1}{\lambda_{j}} \sum_{j=2}^{n-1} \widetilde{W}_{j}^{T}(t) D_{t}^{\alpha} W_{j}(t) \\
= & \sum_{j=2}^{n-1} z_{j}(t)\left[z_{j+1}(t)-W_{j}^{T}(t) \psi\left(\bar{\zeta}_{j}(t)\right)-z_{j-1}(t)-k_{j} z_{j}(t)+\gamma_{j}\left(\bar{\zeta}_{j}(t)\right)\right]+\frac{1}{\lambda_{j}} \sum_{j=2}^{n-1} \widetilde{W}_{j}^{T}(t) D_{t}^{\alpha} W_{j}(t) \\
& -z_{1}(t) \varepsilon_{1}\left(\bar{\zeta}_{1}(t)\right)-k_{1} z_{1}^{2}(t)+z_{1}(t) z_{2}(t) \\
\leq & -\sum_{j=1}^{n-1} k_{j} z_{j}^{2}(t)+\sum_{j=2}^{n-1} z_{j}(t)\left[z_{j+1}(t)-W_{j}^{T}(t) \psi\left(\bar{\zeta}_{j}(t)\right)-z_{j-1}(t)+\gamma_{j}\left(\bar{\zeta}_{j}(t)\right)\right]+\frac{1}{\lambda_{j}} \sum_{j=2}^{n-1} \widetilde{W}_{j}^{T}(t) D_{t}^{\alpha} W_{j}(t) \\
& -\left|z_{1}(t)\right| \bar{\varepsilon}_{1}+z_{1}(t) z_{2}(t) \\
\leq & -\sum_{j=1}^{n-1} k_{j} z_{j}^{2}(t)-z_{n-1}(t) z_{n}(t)+\sum_{j=1}^{n-1} \bar{\varepsilon}_{j}\left|z_{j}(t)\right| .
\end{aligned}
$$

Finally, let

$$
V_{n}(t)=V_{n-1}(t)+\frac{1}{2} z_{n}^{2}(t)+\frac{1}{2 \lambda_{1}} \widetilde{W}_{n}^{T}(t) \widetilde{W}_{n}(t)
$$

Then, after some manipulators, we have

$$
D_{t}^{\alpha} V_{n} \leq-\sum_{j=1}^{n} k_{j} z_{j}^{2}+\sum_{j=1}^{n} \bar{\varepsilon}_{j}\left|z_{j}\right|-\sum_{j=1}^{n} k_{1 j}\left|z_{j}\right| .
$$

Thus, if the parameter $k_{1 j}$ is chosen such that

$$
k_{1 j} \geq \bar{\varepsilon}_{j}
$$

and according to Lemma 1 , the tracking error $z_{j}(t)$ tends to zero asymptotically. This ends the proof of Theorem 1 .

\section{Simulation Studies}

In this part, to check our theoretical derivation, an example will be given. Consider the control problem of fractionalorder Arneodo system, which is given by $[35,36]$

$$
\left\{\begin{array}{l}
D_{t}^{\alpha} \zeta_{1}(t)=f\left(\zeta_{1}(t)\right)+\zeta_{2}(t) \\
D_{t}^{\alpha} \zeta_{2}=f\left(\zeta_{1}(t), \zeta_{2}(t)\right)+\zeta_{3}(t) \\
D_{t}^{\alpha} \zeta_{3}=-b_{1} \zeta_{1}(t)-b_{2} \zeta_{2}(t)-b_{3} \zeta_{3}(t)+b_{4} \zeta_{1}^{3}(t)+u(t)
\end{array}\right.
$$

Let the initial condition be $\zeta_{1}(0)=-2.0, \zeta_{2}(0)=5.01$, and $\zeta_{3}(0)=2.02$. Firstly, if we assume that $f\left(\zeta_{1}(t)\right), f\left(\zeta_{2}(t), \zeta_{2}(t)\right)$, and $u(t)$ are not considered and let $b_{1}=5.50, b_{2}=3.50$, $b_{3}=0.80, b_{4}=1.01$, and $\alpha=0.935$, the chaotic behavior of the system (35) is as given in Figure 2.

Let $\quad f\left(\zeta_{1}(t)\right)=4.5 \zeta_{1} e^{\zeta_{1}^{2}(t)}, f\left(\zeta_{1}(t), \zeta_{2}(t)\right)=3 \zeta_{1}^{2}(t)+$ $\zeta_{2}^{3}(t), \quad k_{1}=k_{2}=k_{11}=k_{12}=0.95$, and $\lambda_{1}=\lambda_{5}=25$. Define the referenced signal $\zeta_{d}(t)$ as

$$
\begin{aligned}
& D_{t}^{\alpha} \zeta_{d}(t)=\zeta_{r}(t) \\
& D_{t}^{\alpha} \zeta_{d}(t)=25 \zeta_{d}(t)-9 \zeta_{r}(t)+25 \zeta_{c}(t)
\end{aligned}
$$

where $\zeta_{c}(t)$ is given by 


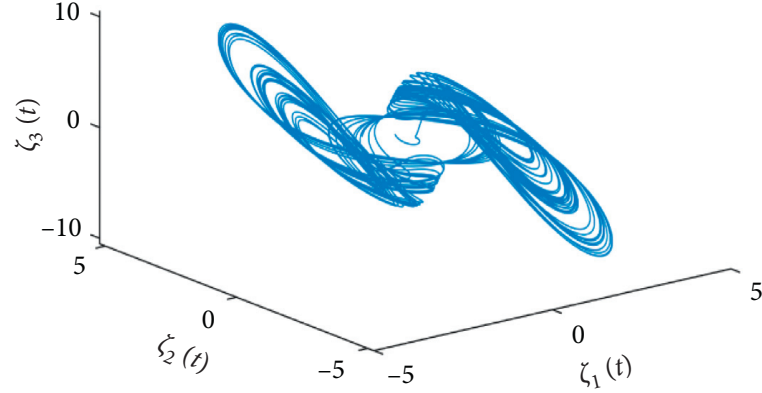

(a)

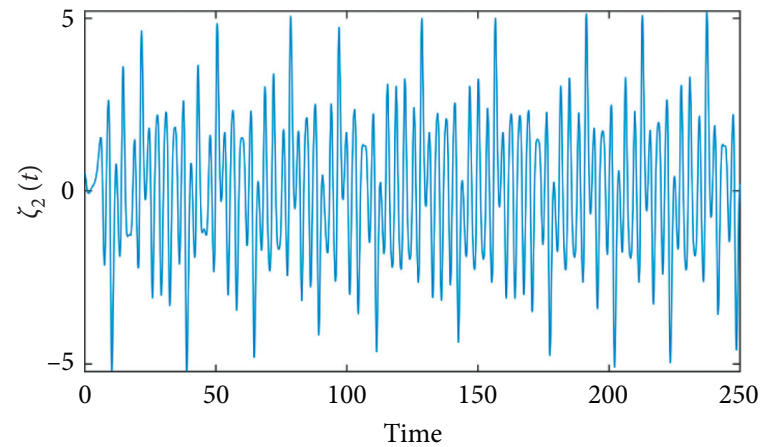

(c)

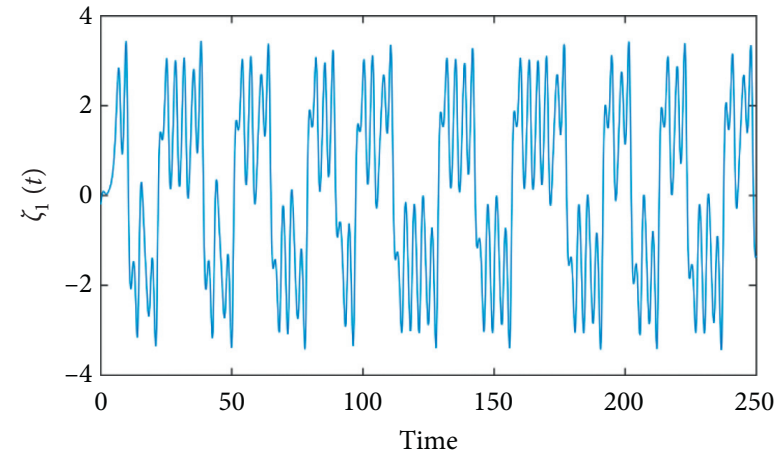

(b)

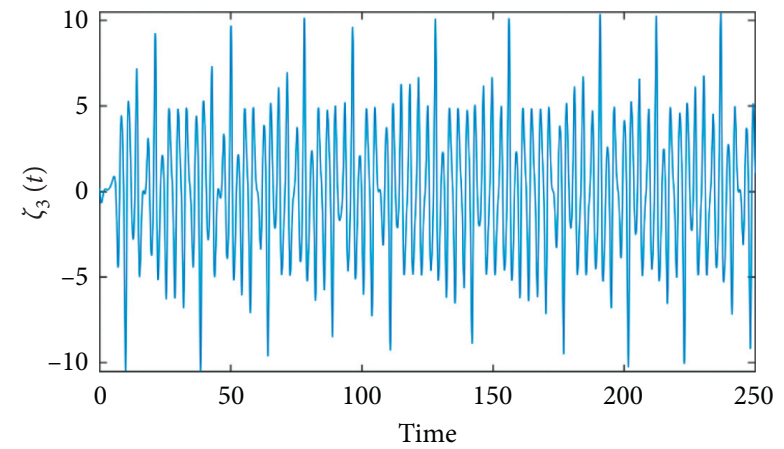

(d)

Figure 2: The chaotic behavior of (35) in (a) $\zeta_{1}-\zeta_{2}-\zeta_{3}$; (b) $\zeta_{1}(t)$; (c) $\zeta_{2}(t)$; and (d) $\zeta_{3}(t)$.

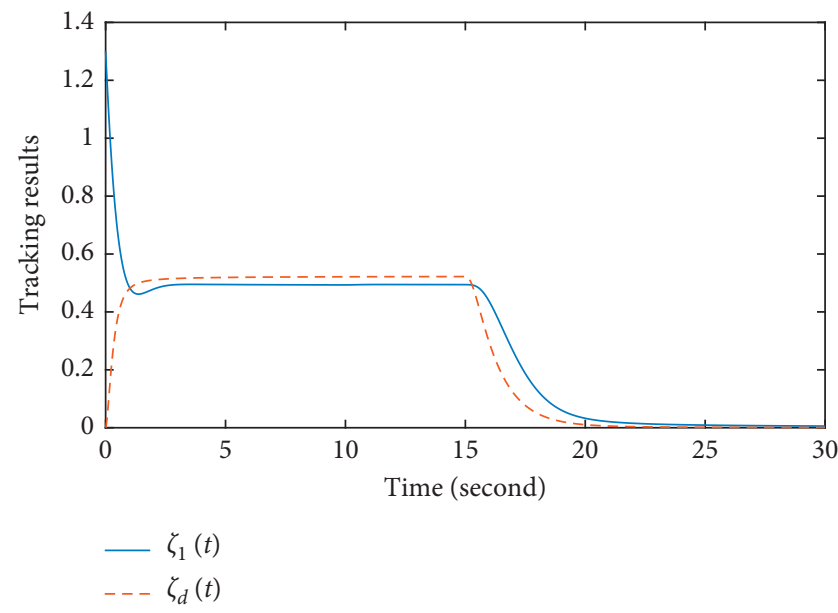

Figure 3: Simulation results.

$$
\zeta_{c}(t)= \begin{cases}\frac{\pi}{6}, & t<15, \\ \frac{\pi e^{-t-15}}{6}, & t \geq 15 .\end{cases}
$$

In the simulation, we should use 3 neural networks, whose nodes are 9, 81, and 729, respectively. The simulation results are shown in Figures 3-7 . Figure 3 mainly depicts the performance of signal $\zeta_{1}(t)$ tracking the signal $\zeta_{d}(t)$ which is defined by (36) and (37). From this figure,

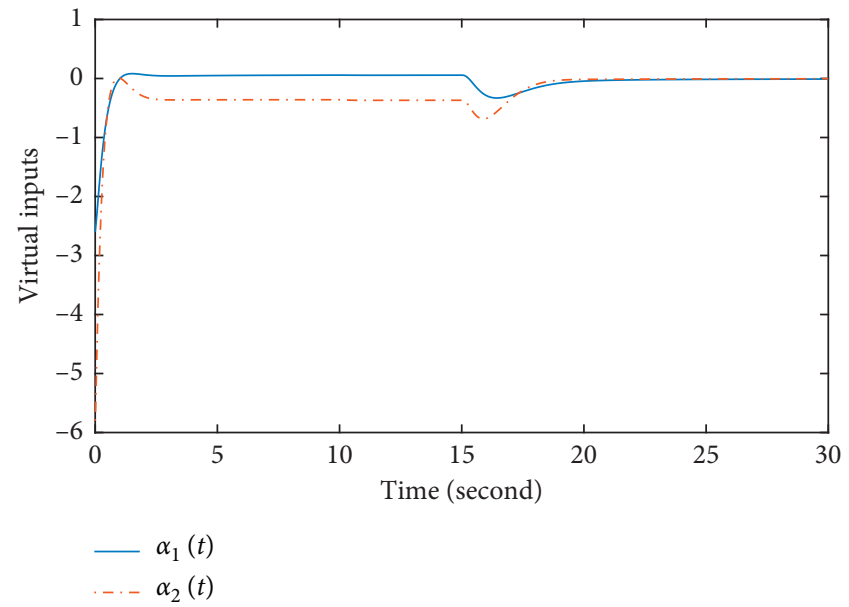

Figure 4: Virtual inputs.

we can see that in the first 15 seconds, that is, when $\zeta_{d}(t)$ is a constant, the tracking performance is good. When the time $t$ is more than 15 seconds, the reference signal $\zeta_{d}(t)$ has a sudden change, but after control, the output of the system can track $\zeta_{d}(t)$ in a very short time. This also shows that the method has very good robustness. Figure 4 gives the virtual inputs $\alpha_{1}(t)$ and $\alpha_{1}(t)$. Figure 5 depicts the final controller $u(t)$. From the above two pictures, we can see that all the inputs are smooth. Figure 6 shows the tracking errors $z_{1}(t), z_{2}(t)$, and $z_{3}(t)$. Finally, Figure 7 depicts the norm of $W_{1}(t), W_{2}(t)$, and $W_{3}(t)$. Generally speaking, our 


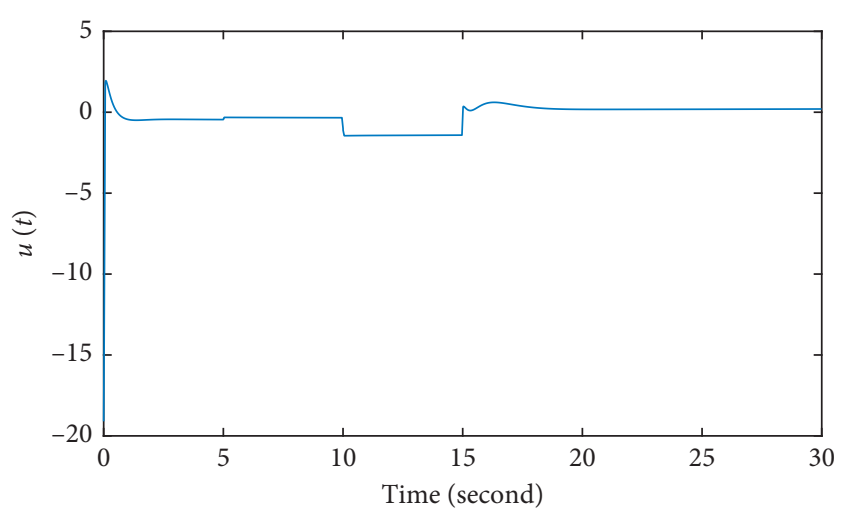

Figure 5: Controller.

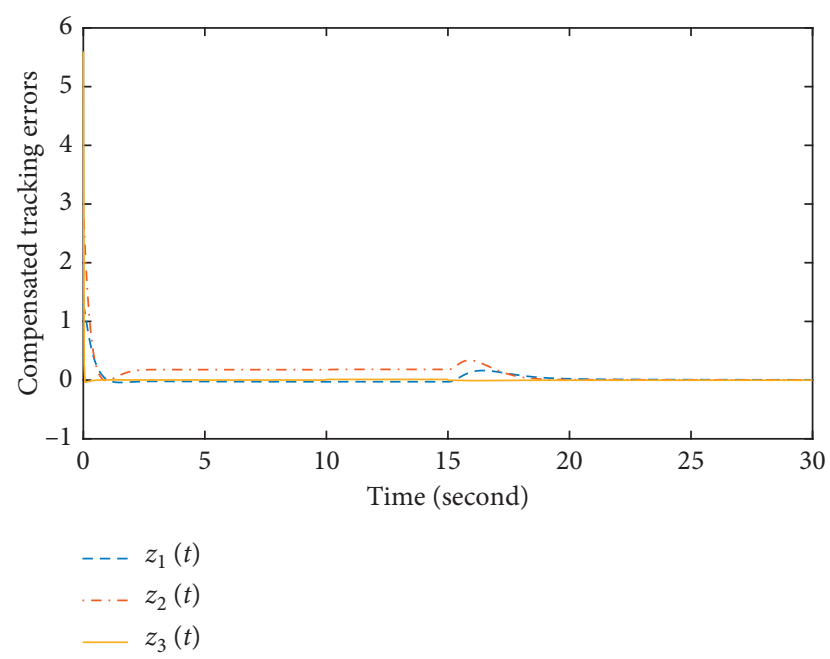

FIGURE 6: $z_{1}(t), z_{2}(t)$, and $z_{3}(t)$.

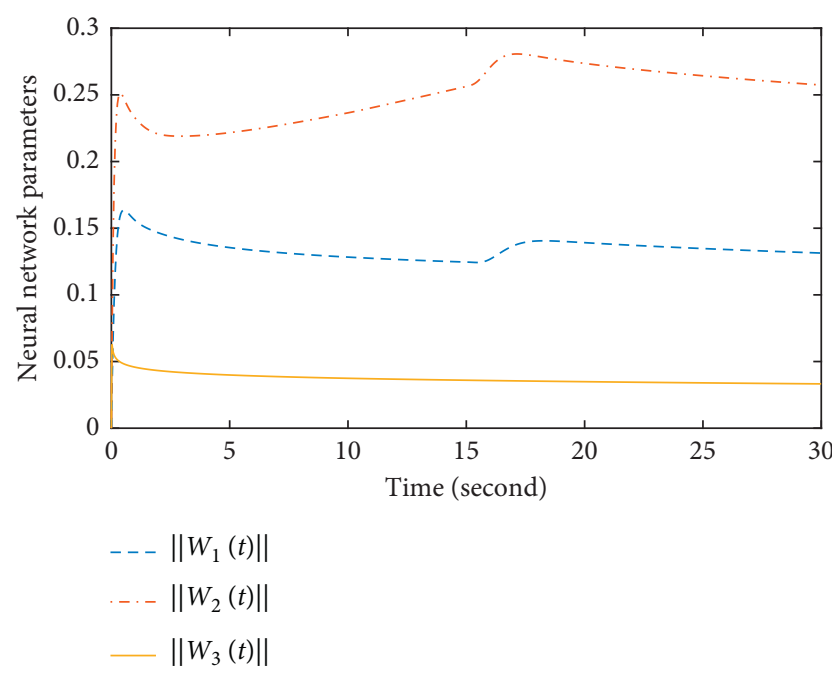

Figure 7: $z_{1}(t), z_{2}(t)$, and $z_{3}(t)$.

method has good tracking performance, can ensure the tracking error convergence quickly, and has good robustness.

\section{Conclusion}

This paper concerns the control problem uncertain fractional-order nonlinear systems by using neural network backstepping technique. First, in the backstepping design, all virtual control inputs' fractional-order derivatives are approximated together with the system uncertainties by using 3-layer neural networks. By using this method, we need not calculate the fractional-order derivative of virtual input repeatedly and thus the problem of "explosion of terms" is overcome. To handle the approximation error, a robust term is needed. Finally, simulation studies illustrate the effectiveness of the proposed method. Noting that the nonlinear input is not considered in this work, how to solve this problem is one of our future research directions.

\section{Data Availability}

All datasets generated for this study are included in the manuscript.

\section{Conflicts of Interest}

The authors declare that there are no conflicts of interest regarding the publication of this paper.

\section{Acknowledgments}

This work was supported by the National Natural Science Foundation of China (Grant no. 11771406) and the 2017 Natural Science Foundation Cooperation Agreement Project (Grant no. 60870).

\section{References}

[1] I. Kanellakopoulos, P. V. Kokotovic, and A. S. Morse, "A toolkit for nonlinear feedback design," Systems \& Control Letters, vol. 18, no. 2, pp. 83-92, 1992.

[2] Z. Jia, J. Yu, Y. Mei, Y. Chen, Y. Shen, and X. Ai, "Integral backstepping sliding mode control for quadrotor helicopter under external uncertain disturbances," Aerospace Science and Technology, vol. 68, pp. 299-307, 2017.

[3] X. Shi, Y. Cheng, C. Yin, S. Dadras, and X. Huang, "Design of fractional-order backstepping sliding mode control for quadrotor UAV," Asian Journal of Control, vol. 21, no. 1, pp. 156-171, 2019.

[4] H. Liu, Y. Pan, S. Li, and Y. Chen, "Adaptive fuzzy backstepping control of fractional-order nonlinear systems," IEEE Transactions on Systems, Man, and Cybernetics: Systems, vol. 47, no. 8, pp. 2209-2217, 2017.

[5] S. A. Moezi, E. Zakeri, and M. Eghtesad, "Optimal adaptive interval type-2 fuzzy fractional-order backstepping sliding mode control method for some classes of nonlinear systems," ISA Transactions, vol. 93, pp. 23-39, 2019.

[6] L. An and G.-H. Yang, "Decentralized adaptive fuzzy secure control for nonlinear uncertain interconnected systems against intermittent dos attacks," IEEE Transactions on Cybernetics, vol. 49, no. 3, pp. 827-838, 2018.

[7] N. Aguila-Camacho, M. A. Duarte-Mermoud, and J. A. Gallegos, "Lyapunov functions for fractional order systems," Communications in Nonlinear Science and $\mathrm{Nu}$ merical Simulation, vol. 19, no. 9, pp. 2951-2957, 2014. 
[8] Y. Li, Y. Chen, and I. Podlubny, "Mittag-leffler stability of fractional order nonlinear dynamic systems," Automatica, vol. 45, no. 8, pp. 1965-1969, 2009.

[9] Y. Wei, J. Wang, T. Liu, and Y. Wang, "Sufficient and necessary conditions for stabilizing singular fractional order systems with partially measurable state," Journal of The Franklin Institute, vol. 356, no. 4, pp. 1975-1990, 2019.

[10] S. Mirzajani, M. P. Aghababa, and A. Heydari, "Adaptive T-S fuzzy control design for fractional-order systems with parametric uncertainty and input constraint," Fuzzy Sets and Systems, vol. 365, pp. 22-39, 2019.

[11] M. Tavazoei and M. H. Asemani, "On robust stability of incommensurate fractional-order systems," Communications in Nonlinear Science and Numerical Simulation, vol. 90, Article ID 105344, 2020.

[12] A. J. Muñoz-Vázquez, J. D. Sánchez-Torres, M. Defoort, and S. Boulaaras, "Predefined-time convergence in fractionalorder systems," Chaos, Solitons \& Fractals, vol. 143, Article ID 110571, 2021.

[13] Y. Zhou, H. Liu, J. Cao, and S. Li, "Composite learning fuzzy synchronization for incommensurate fractional-order chaotic systems with time-varying delays," International Journal of Adaptive Control and Signal Processing, vol. 33, no. 12, pp. 1739-1758, 2019.

[14] Y. Zhou, H. Wang, and H. Liu, "Generalized function projective synchronization of incommensurate fractional-order chaotic systems with inputs saturation," International Journal of Fuzzy Systems, vol. 21, no. 3, pp. 823-836, 2019.

[15] K. Sun, S. Mou, J. Qiu, T. Wang, and H. Gao, "Adaptive fuzzy control for nontriangular structural stochastic switched nonlinear systems with full state constraints," IEEE Transactions on Fuzzy Systems, vol. 27, no. 8, pp. 1587-1601, 2018.

[16] L. Wang, H. Li, Q. Zhou, and R. Lu, "Adaptive fuzzy control for nonstrict feedback systems with unmodeled dynamics and fuzzy dead zone via output feedback," IEEE Transactions on Cybernetics, vol. 47, no. 9, pp. 2400-2412, 2017.

[17] Y.-J. Liu, Q. Zeng, S. Tong, C. L. P. Chen, and L. Liu, "Adaptive neural network control for active suspension systems with time-varying vertical displacement and speed constraints," IEEE Transactions on Industrial Electronics, vol. 66, no. 12, pp. 9458-9466, 2019.

[18] Z. Zhao, J. Shi, X. Lan, X. Wang, and J. Yang, “Adaptive neural network control of a flexible string system with non-symmetric dead-zone and output constraint," Neurocomputing, vol. 283, pp. 1-8, 2018.

[19] S. Luo, F. L. Lewis, Y. Song, and H. M. Ouakad, "Accelerated adaptive fuzzy optimal control of three coupled fractionalorder chaotic electromechanical transducers," IEEE Transactions on Fuzzy Systems, vol. 11 page, 2020.

[20] Z. Ma and H. Ma, "Adaptive fuzzy backstepping dynamic surface control of strict-feedback fractional-order uncertain nonlinear systems," IEEE Transactions on Fuzzy Systems, vol. 28, no. 1, pp. 122-133, 2019.

[21] S. Ha, H. Liu, S. Li, and A. Liu, "Backstepping-based adaptive fuzzy synchronization control for a class of fractional-order chaotic systems with input saturation," International Journal of Fuzzy Systems, vol. 21, no. 5, pp. 1571-1584, 2019.

[22] Y. Wei, D. Sheng, Y. Chen, and Y. Wang, "Fractional order chattering-free robust adaptive backstepping control technique," Nonlinear Dynamics, vol. 95, no. 3, pp. 2383-2394, 2019.

[23] M. K. Shukla and B. B. Sharma, "Control and synchronization of a class of uncertain fractional order chaotic systems via adaptive backstepping control," Asian Journal of Control, vol. 20, no. 2, pp. 707-720, 2018.

[24] S. Han, "Fractional-order command filtered backstepping sliding mode control with fractional-order nonlinear disturbance observer for nonlinear systems," Journal of the Franklin Institute, vol. 357, no. 11, pp. 6760-6776, 2020.

[25] A. A. Jafari, S. M. A. Mohammadi, and M. H. Naseriyeh, "Adaptive type-2 fuzzy backstepping control of uncertain fractional-order nonlinear systems with unknown deadzone," Applied Mathematical Modelling, vol. 69, pp. 506-532, 2019.

[26] Z. Anjum and Y. Guo, "Finite time fractional-order adaptive backstepping fault tolerant control of robotic manipulator," International Journal of Control, Automation and Systems, vol. 19, no. 1, pp. 301-310, 2021.

[27] Z. Yu, Y. Zhang, B. Jiang et al., "Nussbaum-based finite-time fractional-order backstepping fault-tolerant flight control of fixed-wing UAV against input saturation with hardware-inthe-loop validation," Mechanical Systems and Signal Processing, vol. 153, Article ID 107406, 2021.

[28] H. Delavari and H. Heydarinejad, "Fractional-order backstepping sliding-mode control based on fractional-order nonlinear disturbance observer," Journal of Computational and Nonlinear Dynamics, vol. 13, no. 11, 2018.

[29] M. Feindt and U. Kerzel, "The neurobayes neural network package," Nuclear Instruments and Methods in Physics Research Section A: Accelerators, Spectrometers, Detectors and Associated Equipment, vol. 559, no. 1, pp. 190-194, 2006.

[30] O. I. Abiodun, A. Jantan, A. E. Omolara, K. V. Dada, N. A. Mohamed, and H. Arshad, "State-of-the-art in artificial neural network applications: a survey," Heliyon, vol. 4, no. 11, Article ID e00938, 2018.

[31] T. Vijayakumar, "Comparative study of capsule neural network in various applications," Journal of Artificial Intelligence and Capsule Networks, vol. 1, no. 1, pp. 19-27, 2019.

[32] A. H. Elsheikh, S. W. Sharshir, M. Abd Elaziz, A. E. Kabeel, W. Guilan, and Z. Haiou, "Modeling of solar energy systems using artificial neural network: a comprehensive review," Solar Energy, vol. 180, pp. 622-639, 2019.

[33] W. He and Y. Dong, "Adaptive fuzzy neural network control for a constrained robot using impedance learning," IEEE Transactions on Neural Networks and Learning Systems, vol. 29, no. 4, pp. 1174-1186, 2017.

[34] W. He, Z. Yan, C. Sun, and Y. Chen, "Adaptive neural network control of a flapping wing micro aerial vehicle with disturbance observer," IEEE Transactions on Cybernetics, vol. 47, no. 10, pp. 3452-3465, 2017.

[35] J. G. Lu, "Chaotic dynamics and synchronization of fractional-order Arneodo's systems," Chaos, Solitons \& Fractals, vol. 26, no. 4, pp. 1125-1133, 2005.

[36] D. A. Yousri, A. M. AbdelAty, L. A. Said, A. S. Elwakil, B. Maundy, and A. G. Radwan, "Parameter identification of fractional-order chaotic systems using different meta-heuristic optimization algorithms," Nonlinear Dynamics, vol. 95, no. 3, pp. 2491-2542, 2019. 